\title{
Expression of Plectin-1 and Trichohyalin in Human Tongue Cancer Cells
}

\author{
Isao Tamura ${ }^{1}$, Katsura Ueda ${ }^{1}$, Tetsunari Nishikawa ${ }^{2}$, Aiko Kamada ${ }^{3}$, Tomoharu Okamura ${ }^{4}$, \\ Yoshifumi Matsuda1, Kentaro Ueno', Yoshihiro Yoshikawa ${ }^{3}$, Eisuke Domae ${ }^{3}$, Kazuya Tominaga ${ }^{4}$, \\ Shunji Kumabe1, Takashi Ikeo ${ }^{3}$, Akio Tanaka ${ }^{5}$
}

\author{
${ }^{1}$ Department of Oral Anatomy, School of Dentistry, Osaka Dental University, Hirakata, Japan \\ ${ }^{2}$ Department of Innovation in Dental Education, School of Dentistry, Osaka Dental University, Hirakata, Japan \\ ${ }^{3}$ Department of Biochemistry, School of Dentistry, Osaka Dental University, Hirakata, Japan \\ ${ }^{4}$ Department of Oral Pathology, School of Dentistry, Osaka Dental University, Hirakata, Japan \\ ${ }^{5}$ Department of Pathology, School of Dentistry, Osaka Dental University, Hirakata, Japan \\ Email: tamura@cc.osaka-dent.ac.jp
}

How to cite this paper: Tamura, I., Ueda, K., Nishikawa, T., Kamada, A., Okamura, T., Matsuda, Y., Ueno, K., Yoshikawa, Y., Domae, E., Tominaga, K., Kumabe, S., Ikeo, T. and Tanaka, A. (2018) Expression of Plectin-1 and Trichohyalin in Human Tongue Cancer Cells. Open Journal of Stomatology, 8, 196-204.

https://doi.org/10.4236/ojst.2018.86019

Received: April 25, 2018

Accepted: June 12, 2018

Published: June 15, 2018

Copyright (c) 2018 by authors and Scientific Research Publishing Inc. This work is licensed under the Creative Commons Attribution International License (CC BY 4.0).

http://creativecommons.org/licenses/by/4.0/

\section{(c) (i) Open Access}

\begin{abstract}
In basal squamous cells, plectin-1 interacts with intermediate filaments, whereas trichohyalin, which is distributed primarily in the medulla and inner root sheath cells of human hair follicles, plays a role in strengthening cells during keratinization. Although both cytoskeletal proteins occur in trace amounts in human tongue epithelial cells, there are minimal data on their expression in human tongue primary cancer cells. We therefore investigated the expression of plectin-1 and trichohyalin in human tongue epithelial cell line (DOK) and tongue cancer cell line (BICR31) using western blotting and FITC-labeled immunocytochemistry techniques. DOK and BICR31 cells were cultivated to subconfluence in Dulbecco's Modified Eagle's Medium containing $0.4 \mu \mathrm{g} / \mathrm{ml}$ of hydrocortisone and $10 \%$ fetal bovine serum, and the levels of trichohyalin and plectin-1 were determined by western blot analysis and immunocytochemical staining. Trichohyalin expression was clearly observed, with no differences between DOK and BICR31 cells. Although DOK cells expressed trace levels of plectin-1, obvious plectin-1 bands were detected in western blot analyses of BICR31 cells. Immunocytochemical staining revealed that trichohyalin and plectin-1 localize in the cytoplasm. Trichohyalin was diffusely distributed in both cell lines, and colocalization of trichohyalin and cytokeratin $1 / 10$ was observed in almost all BICR31 cells. There were no correlations between western blot and immunocytochemical data for trichohyalin. Conversely, correlations in immunochemical reactions for plectin-1 were observed. Most DOK cells showed no localization of plectin-1, but strong reactions were detected in the cytoplasm of BICR31 cells. These results indicate that trichohyalin is expressed by cancerous tongue epithelial cells during various stages of malignancy and that plectin-1 provides an index of malignancy.
\end{abstract}




\section{Keywords}

Tongue Cancer, Plectin-1, Trichohyalin, Diagnosis

\section{Introduction}

Cancer cells are characterized by phenotypic and biological heterogeneity. Similar to epithelial-to-mesenchymal transition (EMT), the ability of cancer cells to infiltrate tissues and metastasize during carcinogenesis is associated with changes in the expression of cell structural and adhesion molecules as well as matrix metalloproteases [1] [2] [3]. Accordingly, investigations into the changes in expression of these molecules would be useful in developing diagnostic methods and determining prognosis.

Proteins of the plakin family play important roles in ensuring the integrity of epithelial, muscle, and nerve cells [4]. Plectin-1, which is $500-\mathrm{kDa}$ cross-linker protein, belongs to the plakin family of cytoskeletal proteins and interacts with intermediate filaments, being localized into cells in some tissues. As plectin-1 functions as the binding protein of hemi-desmosome bullous pemphigoid antigen [2] and links to intermediate filaments, it was hypothesized that plectin-1 contributes to cell mobility and cytoskeleton formation [5] [6] [7]. However, details regarding the kinetics of plectin-1 in human oral tissues have not been reported.

Trichohyalin is a protein of approximately $200 \mathrm{kDa}$ that is distributed primarily in the medulla and inner root sheath cells of human hair follicles, and it is believed to play a role in maintaining cell strength during keratinization [8] [9]. Although there is no information on its expression in human oral primary cancer cells, trichohyalin expression has been reported in tongue papilla in normal human oral tissue [10] [11].

As plectin-1 and trichohyalin interact with intermediate filaments or actin filaments, it is reasonable to hypothesize that they play roles in the formation of invadopodia and/or filopodia. However, there is little information available regarding their expression in human tongue primary cancer cells.

In the present study, we investigated the expression of plectin-1 and trichohyalin in DOK human tongue epithelial cells [12] and BICR31 tongue cancer cells [13] using western blotting and FITC-labeled immunocytochemistry techniques.

\section{Materials and Methods}

\subsection{Cell Culture}

DOK and BICR31 cells (Figure 1) were purchased from Health Protection Agency (Wiltshire, UK). Cells were cultured in Dulbecco's Modified Eagle Medium (DMEM) supplemented with $2 \mathrm{mM} \mathrm{L-glutamine,} 100 \mu \mathrm{g} / \mathrm{mL}$ streptomycin, 100 $\mathrm{IU} / \mathrm{mL}$ penicillin, $0.4 \mu \mathrm{g} / \mathrm{mL}$ hydrocortisone, and $10 \%$ fetal bovine serum in a 
humidified atmosphere containing $5 \% \mathrm{CO}_{2}$ in air at $37^{\circ} \mathrm{C}$.

\subsection{Western Blot Analyses}

DOK and BICR31 cells were cultivated in $25 \mathrm{~cm}^{2}$ tissue culture flasks and then lysed. The cell lysates were solubilized in SDS-PAGE sample buffer, and protein concentration was determined using the bicinchoninic acid method [14]. Equal amounts of protein samples were fractionated and then transferred electrophoretically onto PVDF membranes [15]. After blocking with $1 \%$ bovine serum albumin (BSA) at $4^{\circ} \mathrm{C}$ for overnight and incubation with antibodies against plectin-1or trichohyalin (Table 1 ) at $4^{\circ} \mathrm{C}$ for overnight, the membranes were labeled using the streptavidin-biotin method [16]. Protein bands were then visualized by subsequent exposure of the membranes on X-ray film, with the molecular size of proteins, specificity of antibodies, and expression of each protein confirmed by western blot analysis.

\subsection{Immunocytochemical Analyses}

DOK and BICR31 cells were seeded at a density of 1000 cells/well in chamber slides and incubated in DMEM, as previously described. After cells were fixed with cold methanol for $20 \mathrm{~min}$, non-specific reactions were blocked with phosphate-buffered saline (PBS) containing 1\% BSA. Treated cells were incubated with the previously described antibodies (Table 1), followed by RITC-conjugated anti-immunoglobulins (DAKO A/S) for cytokeratins or FITC-conjugated anti-immunoglobulins (DAKO $\mathrm{A} / \mathrm{S}$ ) for plectin-1 and trichohyalin. All fluorescence images were finally observed using an all-in-one fluorescent microscopy system (BZ-9000; Keyence Japan, Osaka, Japan). Samples incubated with PBS instead of primary antibody were used as negative controls.

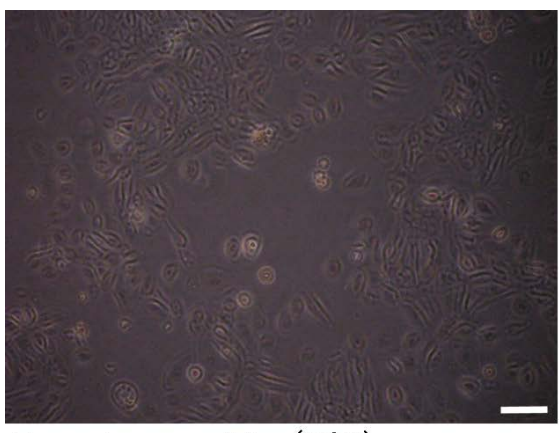

Human tongue cell line (DOK)

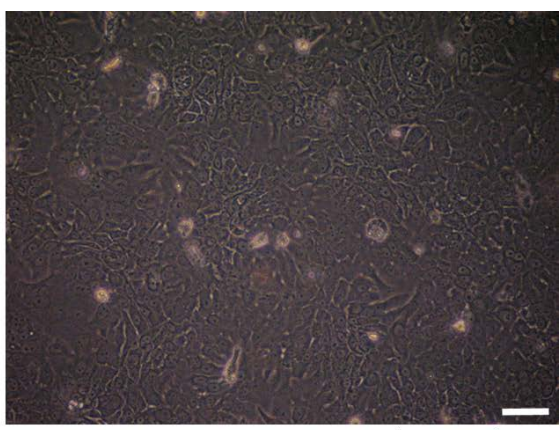

Human tongue cancer cell line (BICR31)

Figure 1. Phase contrast microscopy images $(\times 40$, bar $=125 \mu \mathrm{m})$ of experimental cells.

Table 1. The list of antibodies.

\begin{tabular}{cccc}
\hline & Clone & Cytology & Blotting \\
\hline Plectin 1 & C-20 & $1: 200$ & $1: 400$ \\
Trichohyalin & K-16 & $1: 200$ & $1: 400$ \\
Cytokeratin 1/10 & LH1 & $1: 100$ & - \\
\hline
\end{tabular}




\section{Results}

\subsection{Western Blot Profiles}

Western blot analyses confirmed the molecular weight and expression of each protein (Figure 2). Anti-plectin-1 antibody reacted in the extreme high-molecular-weight region with BICR31 cells (Figure 2, arrow) but not DOK cells. Both DOK and BICR31 cells expressed trichohyalin, which showed a 200-kDa band.

\subsection{Immunocytochemical Findings}

On immunocytochemical staining, plectin-1 (Figure 3) and trichohyalin (Figure 4) were localized. There were correlations between western blot and immunocytology results for plectin-1. No localization of plectin-1 was observed in most DOK cells, but strong immunoreaction was detected in BICR31 cells. Additionally, plectin-1 and CK 1/10 colocalized widely in BICR31 cells (Figure 3, merge). Conversely, no immunochemical correlations were observed for trichohyalin. Anti-trichohyalin antibody reacted in thecytoplasm and partially in the nuclear region (Figure 4). Trichohyalin was diffusely distributed in DOK cells, and the immunoreaction was weak. The anti-trichohyalin antibody reacted stronger in BICR31 cells than DOK cells.

\section{Discussion}

Plectin-1 was the first cytoskeletal component discovered to connect intermediate filaments in epithelial cells of the small intestine, liver, and urinary bladder. In addition, plectin-1 is expressed in muscle and nerve cells, and it is hypothesized that the protein is involved in complex functions [4] [17] because of its distribution in crosslinks of microtubules and actin filaments. It is generally known that plectin-1 is not expressed in normal tongue cells [17], and we observed almost no plectin-1 expression in DOK cells, a human tongue epithelial cell line. Therefore, it is assumed that tongue epithelial cells have a "thin" cell structure and that the cytoskeleton is maintained by an intercellular component. Plectin-1 knock down in human liver cells treated with plectin-1 siRNA was shown to induce a decrease in the expression of CK18, the disorganization of intermediate filaments, and an increase in actin fibers [18]. Accordingly, chief cytoskeleton components in human tongue epithelial cells could be other molecules such as actin, tubulin, and heat shock proteins [19]. By contrast, BICR31 human tongue cancer cells showed clear expression of plectin-1. Similarly, negative immunoreaction showed that plectin-1 is not expressed in normal human pancreas cells, but an increase in plectin-1 expression was reported during pancreatic ductal carcinogenesis and metastasis to the lymph nodes, liver, and peritoneum [20]. These data suggest that plectin-1 could serve as a tumor marker in tongue and pancreatic tissues. Additionally, the observation that ablation of plectin-1 in human colon carcinoma cells treated with siRNA impairs their growth, migration, and adhesion [21] suggests that plectin-1 is involved in malignant transformation of epithelial cells. 

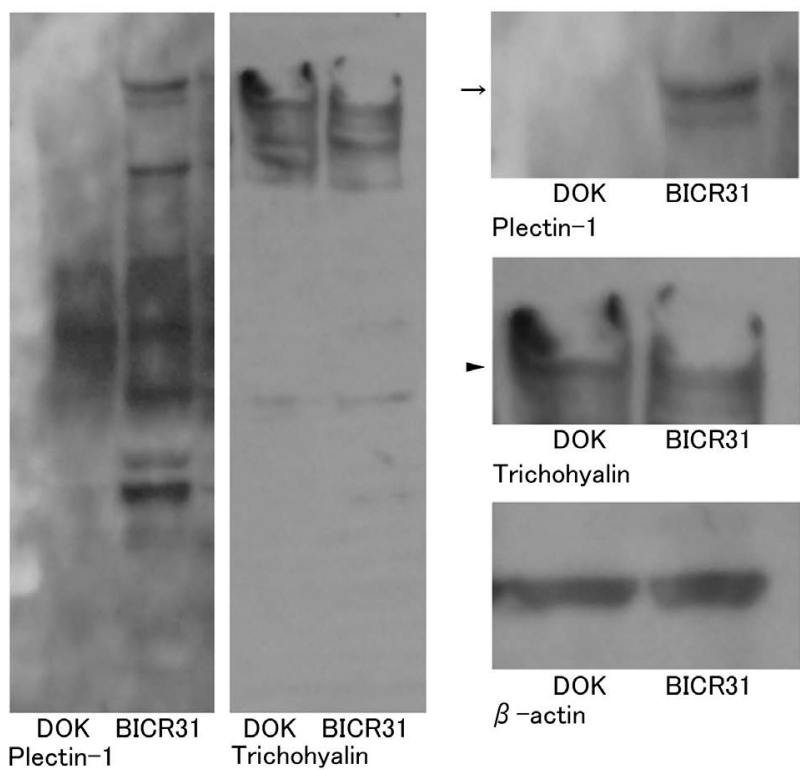

Figure 2. Western blotting analyses. BICR31 cells showed high expression of plectin-1 (arrow). No differences between DOK and BICR31 cells were observed in terms of trichohyalin expression (arrowhead).
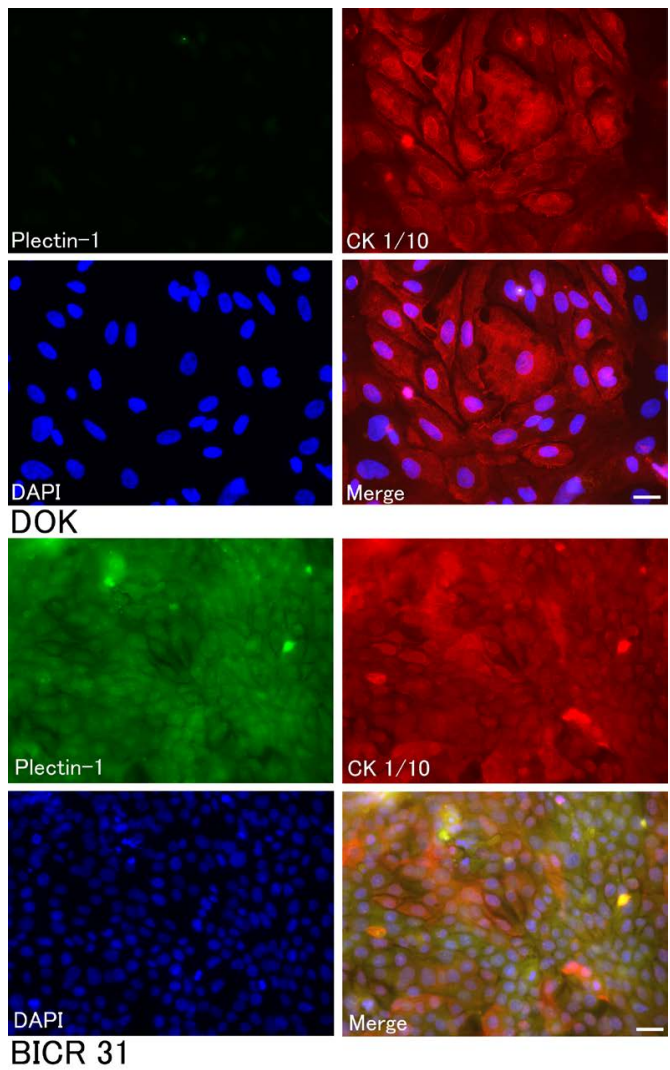

Figure 3. Immunocytochemical observations of plectin-1 expression in experimental cells $(\times 200$, bar $=25 \mu \mathrm{m})$. Although almost no plectin-1 expression was observed in DOK cells, BICR31 cells clearly expressed plectin-1. 

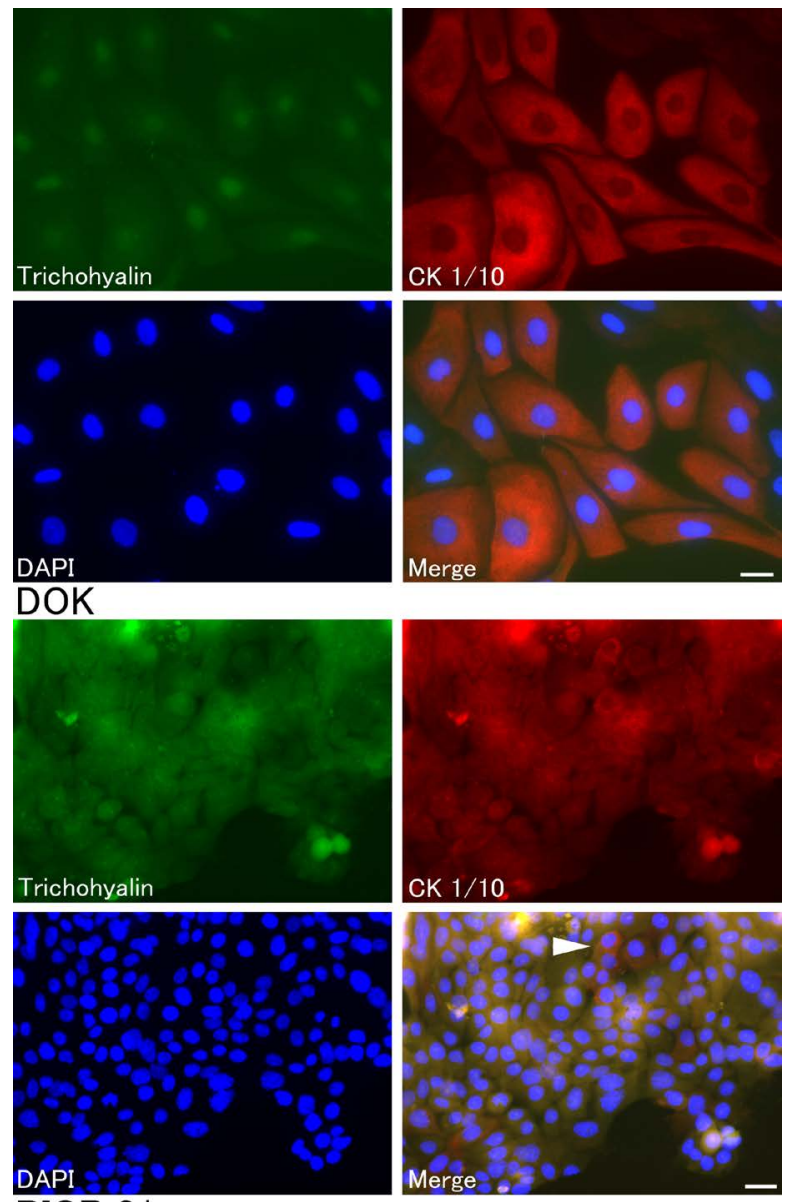

\section{BICR 31}

Figure 4. Immunocytochemical observations of trichohyalin expression in experimental cells $(\times 200$, bar $=25$ $\mu \mathrm{m})$. In immunocytochemical staining, trace or diffuse localization of trichohyalin was observed in all compartments of DOK cells. However, trichohyalin expression was widely distributed in BICR31 cells, and some colocalization of trichohyalin and CK 1/10 was observed (white arrowhead in merged image).

Immunoelectron microscopy studies recently revealed that trichohyalin, which localizes near parallel bundles of intermediate filaments in a manner that promotes the aggregation and lateral alignment of filament bundles [22], exhibits up regulated expression in human tongue epithelia [23] and skin epidermal diseases, including squamous cell carcinoma [24]. In the present study, expression of trichohyalin was not related to the malignancy of cells and did not change over succeeding generations of cells.

Plectin-1 and trichohyalin are known to connect intermediate filaments of intact epithelial cells [4] [22]. However, there is little information regarding the mode of their interaction in human tongue primary cancer cells. Although we performed co-immunoprecipitation analyses for CK 1/10 followed by western blot analyses, we did not obtain clear, reproducible results in immunoreactions 
for plectin-1 and trichohyalin (data not shown). A recent study using a sensitive fluorescent protein-binding assay showed that the C-terminus of plectin-1 interacts predominantly with dimeric CK $1 / 10$ or CK 5/14 rather than monomeric cytokeratins [25]. Accordingly, the results of segmentation analyses might show that plectin-1 or trichohyalin and CK do not interact in the presence of non-ionic surfactants and that trichohyalin and CK 1/10 do not form chemical bonds.

The results of the present study indicate that trichohyalin is expressed by cancerous tongue epithelial cells during various stages of malignancy and that plectin-1 is an index of malignancy.

\section{Acknowledgements}

This study was performed using the Morphological Research Facilities, Low-Temperature Facilities, Photograph-Processing Facilities, Analytical Instrument Facilities, and Dental Bioscience Facilities I of the Institute of Dental Research, Osaka Dental University.

\section{References}

[1] Sakaki, T., Tamura, I., Kadota, H. and Kakudo, K. (2003) Changing Expression of E- and P-Cadherin during Rat Tongue Carcinogenesis Induced by 4-Nitroquinoline 1-Oxide. Journal of Oral Pathology \& Medicine, 32, 530-537. https://doi.org/10.1034/j.1600-0714.2003.00174.x

[2] Tamura, I., Sakaki, T., Chaqour, B., Howard, P.S., Ikeo, T. and Macarak, E.J. (2003) Correlation of P-Cadherin and $\beta$-Catenin Expression and Phosphorylation with Carcinogenesis in Rat Tongue Cancer Induced with 4-Nitroquinoline 1-Oxide. Oral Oncology, 39, 506-514. https://doi.org/10.1016/S1368-8375(03)00013-7

[3] Long, H.A., Boczonadi, V., Mclnroy, L., Goldberg, M. and Määttä, A. (2006) Periplakin-Dependent Re-Organisation of Keratin Cytoskeleton and Loss of Collective Migration in Keratin-8-Downregulated Epithelial Sheets. Journal of Cell Science, 119, 5147-5159. https://doi.org/10.1242/jcs.03304

[4] Sonnenberg, A. and Liem, R.K.H. (2007) Plakins in Development and Disease. EXperimental Cell Research, 313, 2189-2203. https://doi.org/10.1016/j.yexcr.2007.03.039

[5] Andrä, K., Nikolic, B., Stöcher, M., Drenckhahn, D. and Wiche, G. (1998) Not Just Scaffolding: Plectin Regulates Actin Dynamics in cultured cells. Genes \& Development, 12, 3442-3451. https://doi.org/10.1101/gad.12.21.3442

[6] Wiche, G. (1998) Role of Plectin in Cytoskeleton Organization and Dynamics. Journal of Cell Science, 111, 2477-2486.

[7] Boczonadi, V., Mclnroy, L., Goldberg, M. and Määttä, A. (2007) Cytolinker Cross-Talk: Periplakin N-Terminus Interacts with Plectin to Regulate Keratin Organisation and Epithelial Migration. Experimental Cell Research, 313, 3579-3591. https://doi.org/10.1016/j.yexcr.2007.07.005

[8] O’Keefe, E.J., Erickson, H.P. and Bennett, V. (1989) Desmoplakin I and Desmoplakin II Purification and Characterization. Journal of Biological Chemistry, 264, 8310-8318.

[9] Tarcsa, E., Marekov, L.N., Andreoli, J., Idler, W.W., Candi, E., Chung, S.I. and Stei- 
nert, P.M. (1997) The Fate of Trichohyalin Sequential Post-Translational Modifications by Peptidyl-Arginine Deiminase and Transglutaminases. Journal of Biological Chemistry, 272, 27893-27901. https://doi.org/10.1074/jbc.272.44.27893

[10] Hamilton, E.H., Sealock, R., Wallace, N.R. and O’Keefe, E.J. (1992) Trichohyalin: Purification from Porcine Tongue Epithelium and Characterization of the Native Protein. Journal of Investigative Dermatology, 98, 881-889. https://doi.org/10.1111/1523-1747.ep12459412

[11] Manabe, M. and O'Guin, W.M. (1994) Existence of Trichohyalin-Keratohyalin Hybrid Granules: Co-Localization of Two Major Intermediate Filament-Associated Proteins in Non-Follicular Epithelia. Differentiation, 58, 65-75. https://doi.org/10.1046/j.1432-0436.1994.5810065.x

[12] Chang, S.E., Foster, S., Betts, D. and Marnock, W.E. (1992) DOK, a Cell Line Established from Human Dysplastic Oral Mucosa, Shows a Partially Transformed Non-Malignant Phenotype. International Journal of Cancer, 52, 896-902. https://doi.org/10.1002/ijc.2910520612

[13] Fitzsimmons, S.A., Ireland, H., Barr, N.I., Cuthbert, A.P., Going, J.J., Newbold, R.F. and Parkinson, E.K. (2003) Human Squamous Cell Carcinomas Lose a Mortality Gene from Chromosome 6q14.3 to q15. Oncogene, 22, 1737-1746. https://doi.org/10.1038/sj.onc.1206295

[14] Smith, P.K., Krohn, R.I., Hermanson, G.T., Mallia, A.K., Gartner, F.H., Provenzano, M.D., Fujimoto, E.K., Goeke, N.M., Olson, B.J. and Klenk, D.C. (1985) Measurement of Protein Using Bicinchoninic Acid. Analytical Biochemistry, 150, 76-85. https://doi.org/10.1016/0003-2697(85)90442-7

[15] Towbin, H., Staehelin, T. and Gordon, J. (1979) Electrophoretic Transfer of Proteins from Polyacrylamide Gels to Nitrocellulose Sheets: Procedure and Some Applications. Proceedings of the National Academy of Sciences of the United States of America, 76, 4350-4354. https://doi.org/10.1073/pnas.76.9.4350

[16] Shi, Z.R., Itzkowitz, S.H. and Kim, Y.S. (1988) A Comparison of Three Immunoperoxidase Techniques for Antigen Detection in Colorectal Carcinoma Tissues. Journal of Histochemistry \& Cytochemistry, 36, 317-322.

https://doi.org/10.1177/36.3.3278057

[17] Wiche, G., Krepler, R., Artlieb, U., Pytela, R. and Denk, H. (1983) Occurrence and Immunolocalization of Plectin in Tissues. The Journal of Cell Biology, 97, 887-901. https://doi.org/10.1083/jcb.97.3.887

[18] Liu, Y.H., Cheng, C.C., Ho, C.C., Chao, W.T., Pei, R.J., Hsu, Y.H., Ho, L.C., Shiu, B.H. and Lai, Y.S. (2011) Plectin Deficiency on Cytoskeletal Disorganization and Transformation of Human Liver Cells in Vitro. Medical Molecular Morphology, 44, 21-26. https://doi.org/10.1007/s00795-010-0499-y

[19] Thiel, U.J.E., Feltens, R., Adryan, B., Gieringer, R., Brochhhausen, C., Schuon, R., Fillies, T., Grus, F., Mann, W.J. and Brieger, J. (2011) Analysis of Differentially Expressed Proteins in Oral Squamous Cell Carcinoma by MALDI-TOF MS. Journal of Oral Pathology \& Medicine, 40, 369-379.

https://doi.org/10.1111/j.1600-0714.2010.00982.x

[20] Bausch, D., Thomas, S., Mino-Kenudson, M., Fernández-del Castillo, C., Bauer, T.W., Williams, M., Warshaw, A.L., Thayer, S.P. and Kelly, K.A. (2011) Plectin-1 as a Novel Biomarker for Pancreatic Cancer. Clinical Cancer Research, 17, 302-309. https://doi.org/10.1158/1078-0432.CCR-10-0999

[21] Mclnroy, L. and Määttä, A. (2011) Plectin Regulates Invasiveness of SW480 Colon Carcinoma Cells and Is Targeted to Podosome-Like Adhesions in an Iso- 
form-Specific Manner. Experimental Cell Research, 317, 2468-2478.

https://doi.org/10.1016/j.yexcr.2011.07.013

[22] O'Guin, W.M. and Manabe, M. (1991) The Role of Trichohyalin in Hair Follicle Differentiation and Its Expression in Nonfollicular Epithelia. Annals of the New York Academy of Sciences, 642, 51-63. https://doi.org/10.1111/j.1749-6632.1991.tb24380.x

[23] Hamilton, E.H., Payne Jr., R.E. and O’Keefe, E.J. (1991) Trichohyalin: Presence in the Granular Layer and Stratum Corneum of Normal Human Epidermis. Journal of Investigative Dermatology, 96, 666-672. https://doi.org/10.1111/1523-1747.ep12470590

[24] Lee, S.C., Lee, J.B., Seo, J.J. and Kim, Y.P. (1999) Expression of Trichohyalin in Dermatological Disorders: A Comparative Study with Involucrin and Filaggrin by Immunohistochemical Staining. Acta Dermato-Venereologica, 79, 122-126. https://doi.org/10.1080/000155599750011336

[25] Bouameur, J.E. Favre, B., Fontao, L., Lingasamy, P., Begré, N. and Borradori, L. (2014) Interaction of Plectin with Keratin 5 and 14: Dependence on Several Plectin Domains and Keratin Quaternary Structure. Journal of Investigative Dermatology, 134, 2776-2783. https://doi.org/10.1038/jid.2014.255 